\title{
Theory requirements for SM Higgs and EW precision physics at the FCC-ee
}

\author{
S. Heinemeyer ${ }^{1,2,3, a}$ (D) S. Jadach ${ }^{4}$, J. Reuter $^{5}$ (D) \\ ${ }^{1}$ IFT (UAM/CSIC), Universidad Autónoma de Madrid, Cantoblanco 8048, Spain \\ 2 Campus of International Excellence UAM+CSIC, Cantoblanco 28049, Madrid, Spain \\ 3 Instituto de Física de Cantabria (CSIC-UC), Santander 39005, Spain \\ ${ }^{4}$ Institute of Nuclear Physics Polish Academy of Sciences, Krakow, Poland \\ 5 DESY, Theory Group, Notkestr. 85, Hamburg 22607, Germany
}

Received: 5 May 2021 / Accepted: 14 August 2021

(C) The Author(s) 2021, corrected publication 2022

\begin{abstract}
High-precision experimental measurements of the properties of the Higgs boson at $\sim 125 \mathrm{GeV}$ as well as electroweak precision observables such as the $W$-boson mass or the effective weak leptonic mixing angle are expected at future $e^{+} e^{-}$colliders such as the FCC-ee. This high anticipated precision has to be matched with theory predictions for the measured quantities at the same level of accuracy. We briefly summarize the status of these predictions within the standard model and of the tools that are used for their determination. We outline how the theory predictions will have to be improved in order to reach the required accuracy, and also comment on the simulation frameworks for the Higgs and EW precision program.
\end{abstract}

\section{Introduction}

With the discovery of the Higgs boson, all possible elements of the standard model (SM) have been experimentally confirmed and tested in great depth. On the other hand, observational evidence for dark matter and the matter-antimatter asymmetry require physics beyond the SM. One promising way to probe such new physics is through precision measurements of the properties of the Higgs boson. A complementary way is to measure electroweak precision (pseudo-)observables (EWPO) with higher precision. These are the avenues pursued by several proposals for a future $e^{+} e^{-}$collider. In particular, the FCC-ee concept is designed to run at $\sqrt{s}=250 \mathrm{GeV}$ as a Higgs factory, and at $\sqrt{s} \sim M_{Z}, 2 M_{W}$ for high-precision EWPO measurements. In this way, it can improve (indirect) probes for new physics by several orders of magnitude compared to existing bounds [1-3].

The anticipated experimental accuracy of an observable has to be matched with a theory prediction of at least the same level of accuracy to make maximum use of the experimental data. For the accurate study of the properties of the Higgs boson, precise predictions for the various partial decay widths, the branching ratios (BRs) and the Higgs-boson production cross sections along with their theoretical uncertainties are indispensable. For the EWPO, on the one hand, the SM prediction has to reach the level of the experimental accuracy. Similarly,

\footnotetext{
a e-mail: Sven.Heinemeyer@cern.ch (corresponding author)
} 
the extraction of these quantities from experimental data must be equally well under control. All these types of uncertainties (various theoretical ones and experimental) must be taken into account when deriving constraints on new physics from the data.

Several sources of theory uncertainties have to be distinguished. The intrinsic uncertainties are due to missing higher orders in the perturbative expansion of the SM (or BSM) prediction for an observable. The parametric uncertainties are due to the imperfect experimental knowledge of the SM input parameters (as well as theory uncertainties induced in their extraction from data). The extraction of a quantity from a cross section or an asymmetry requires the theory prediction of this cross section or asymmetry to at least the same order of precision. ${ }^{1}$

In this paper, the current status and future implications of theory uncertainties on (SM) Higgs-boson physics and EWPO's will be summarized. While we focus on the FCC-ee, they are valid for all future high precision $e^{+} e^{-}$colliders running above the $H Z$ threshold (such as ILC, CLIC, or CEPC). We will use anticipated FCC-ee precisions to illustrate the impact of theory uncertainties. We build on [4], where also a discussion of the determination of the SM input parameters can be found. In addition, we review Monte Carlo (MC) event generator frameworks which are indispensable for simulations in the perturbative and nonperturbative regime, building upon the experience gained from the LEP era [5-7] and the extensive simulations for CEPC [8,9], ILC [10-13] and CLIC [14-16]. Work on dedicated FCC-ee MC samples has started.

\section{Pseudo-observables versus realistic observables}

The quantities that can be directly measured in experiments are cross sections, line shape observables, forward-backward asymmetries, etc., called "realistic observables" $[17,18]$. The obtained results depend on the specific set of experimental cuts; however, detector inefficiencies are removed and kinematic cuts are brought to simplified form using MC event generators. In order to determine idealized quantities like masses, partial widths or couplings, or the effective electroweak mixing angle, from the primarily measured realistic observables, a "QED unfolding" procedure is applied. This procedure involves manipulations like subtracting photon-exchange or interference terms, subtracting box diagram contributions, unfolding higher-order QED or QCD corrections, etc. These secondary quantities are therefore called "pseudo-observables". The Higgs-boson observables and EWPOs on which we focus here are pseudo-observables. It should be stressed that the role of EWPOs is to represent and encapsulate experimental data in the model-independent way. ${ }^{2}$ Fitting EWPOs to the SM and its extensions comes as an independent next step. In order to reach the required theoretical accuracy special care has to be taken in the application of these unfolding procedures-in particular, the procedure of the extraction of EWPOs from data causes certain bias, partial loss of information on physics in the realistic data. The unfolding procedure for the LEP experiments is described in Refs. [17,18]. A discussion of the additional problems in this respect arising in beyond the SM (BSM) models can be found in [19]. In Refs. [17,18], it was proven that the bias induced in the extraction of EWPOs (QED deconvolution) was smaller than the LEP experimental errors. This will not be true in the FCC-ee era where experimental errors will be smaller by up to two orders. The very survival of the technique of the

\footnotetext{
${ }^{1}$ Theoretical predictions for the past electron collider experiments (LEP/SLC) were at least a factor 3 more precise than experimental data.

${ }^{2}$ For instance $\sin ^{2} \theta_{\text {eff }}^{f}$ is not a SM parameter-it parametrizes data on the ratio of the vector to axial couplings in $e^{+} e^{-} \rightarrow f \bar{f}$.
} 
model-independent EWPOs as the bridge between the experiment and the theory at FCC-ee will require significant improvement of the precision of the trivial but large QED effects. Reference [20] covers this issue in a great detail, listing challenges and outlining solutions. In addition, the definition of EWPOs and the algorithm of their extraction will have to be modified. This is elaborated in Sect. 3 in Ref. [21]. Two major changes are anticipated. One is that we may be forced to remove from EWPOs not only QED but also first order pure electroweak corrections, in order to achieve sharper resolution in the search of the BSM effects in the new EWPOs. Another important change will be the increased role of Monte Carlo generators in extracting EWPOs from data-this is because semianalytic programs like ZFITTER and TOPAZ0 of LEP era will not be able to handle precisely enough detector geometry (cutoffs).

\section{Electroweak precision observables}

The most important electroweak precision observables (EWPOs) are related to properties of the $Z$ and $W$ bosons. The $Z$-boson properties are determined from measurements of $e^{+} e^{-} \rightarrow f \bar{f}$ on the $Z$-pole. To isolate the physics of the $Z$-boson, the typical set of pseudoobservables is defined in terms of the de-convoluted cross-section $\sigma_{f}(s)$, where the effect of initial- and final-state photon radiation and from $s$-channel photon and double-boson (box) exchange has been removed. The customary set of pseudo-observables are total and partial $Z$-boson cross sections with their subsequent decay to quarks or leptons, forward-backward and left-right asymmetries, from which the effective weak leptonic mixing angle, $\sin ^{2} \theta_{\text {eff }}^{f}$, is extracted, as well as the $W$-boson mass, $M_{W}$, see Ref. [22]. The expected (pure) experimental precision at FCC-ee of $M_{W}$ and $\sin ^{2} \theta_{\text {eff }}^{\ell}$ is summarized in Table 1 based on Refs. [2,3].

The quantities listed in Table 1 can be predicted within the SM in terms of the input parameters $G_{F}, \alpha\left(M_{Z}\right), \alpha_{s}\left(M_{Z}\right), M_{Z}, M_{H}$ and $m_{t}$. The radiative corrections in these predictions are currently known including complete two-loop corrections In addition, approximate three- and four-loop corrections of $\mathcal{O}\left(\alpha_{t}^{3}\right), \mathcal{O}\left(\alpha_{t}^{2} \alpha_{s}\right), \mathcal{O}\left(\alpha_{t} \alpha_{s}^{2}\right)$ and $\mathcal{O}\left(\alpha_{t} \alpha_{s}^{3}\right)$ are available (with $\alpha_{t}=y_{t}^{2} /(4 \pi)$ and $y_{t}$ being the top Yukawa coupling), see [23] for a review. The theory uncertainties from missing higher-order corrections are given in the third column of Table 1 [24-27]. Also indicated are the main sources for the respective uncertainties. In order to match the FCC-ee precision the theory predictions will have to be improved substantially. It was estimated in $[4,21,28]$ that the intrinsic uncertainties will be reduced by complete $\mathcal{O}\left(\alpha \alpha_{s}^{2}\right)$ corrections, fermionic $\mathcal{O}\left(\alpha^{2} \alpha_{s}\right)$ corrections, double-fermionic $\mathcal{O}\left(\alpha^{3}\right)$ corrections, and leading four-loop corrections enhanced by the top Yukawa coupling, resulting in the projected intrinsic uncertainties shown in column four of Table 1.

A crucial point here is that the determination of the pseudo-observables in Table 1 from experimental data also requires theory input for the removal of initial-state and final-state pho-

Table 1 Estimated precision for the direct determination of $M_{W}$ and $\sin ^{2} \theta_{\text {eff }}^{\ell}$ at FCC-ee [2,3] (column two), current intrinsic uncertainty (column three) and projected future uncertainty when leading three-loop corrections become available (column 4)

\begin{tabular}{lllll}
\hline Quantity & FCC-ee & \multicolumn{2}{l}{ Current intrinsic unc. } & Projected intrinsic unc. \\
\hline$\delta M_{W}[\mathrm{MeV}]$ & 0.5 & 4 & $\left(\alpha^{3}, \alpha^{2} \alpha_{S}\right)$ & 1 \\
$\delta \sin ^{2} \theta_{\text {eff }}^{\ell}\left[10^{-5}\right]$ & 0.6 & 4.5 & $\left(\alpha^{3}, \alpha^{2} \alpha_{S}\right)$ & 1.5 \\
\hline
\end{tabular}


ton radiation and $s$-channel photon exchange and box contributions. The theory uncertainty from missing higher QED orders is estimated to amount to a few times $0.01 \%$ [5, 17, 18,29] for the $Z$-peak cross-section and total width measurements. In order not to be dominated by this uncertainty, it will need to be reduced by about a factor 10 for the FCC-ee. This will require the calculation of non-leading log contributions to $\mathcal{O}\left(\alpha^{3}\right)$ corrections, $\mathcal{O}\left(\alpha^{3} L^{2}\right)$ and $\mathcal{O}\left(\alpha^{4} L^{4}\right)$ contributions (with $\left.L \equiv \log s / m_{e}^{2}\right)$ as well as an improved treatment of fermion pair production from off-shell photons.

The $W$-boson mass will be determined from a threshold scan near the $W$-pair threshold, $\sqrt{s} \approx 161 \mathrm{GeV}$. It is foreseen that the experimental uncertainty at FCC-ee for this measurement is about $0.5 \mathrm{MeV}$ [1-3]. At the point of highest sensitivity, an uncertainty of the cross-section measurement of $0.1 \%$ translates to an uncertainty of $\sim 1.5 \mathrm{MeV}$ on $M_{W}$ [30]. Therefore a theoretical prediction for the process $e^{+} e^{-} \rightarrow 4 f$ with an accuracy of $\Delta \sigma \sim 0.01 \%$ is desirable, including effects of off-shell $W$ bosons, which become important near threshold.

The currently best calculations are based on complete one-loop results for $e^{+} e^{-} \rightarrow$ $4 f$ [31] and partial higher-order effects for the total cross section from an effective field theory framework $[32,33]$. The resulting theory uncertainty on $M_{W}$ is estimated to be about $3 \mathrm{MeV}$ [33]. This result must be improved by complete two-loop corrections to $e^{+} e^{-} \rightarrow W^{+} W^{-}$ and to $W \rightarrow f \bar{f}^{\prime}$ (based on the effective field theory framework). In addition, a more accurate description of initial-state radiation will be important, which includes universal contributions from soft and collinear photon radiation (see [5,17,29] for a review), as well as hard photon radiation. For the latter, a proper matching and merging procedure needs to be employed to avoid double counting [32,34], see [4] for more details. There it was estimated that a theory induced systematic uncertainty of $\Delta M_{W} \lesssim 0.60 \mathrm{MeV}$ can be feasible.

\section{The SM Higgs boson}

\subsection{Higgs-boson production}

The very narrow width of the Higgs boson allows for a factorization of all cross sections with resonant Higgs bosons into production and decay parts to very high precision if the Higgs boson can be fully reconstructed. In this case, finite-width effects and off-shell contributions are of relative size $\Gamma_{H} / M_{H} \sim 0.00003$ and thus not relevant. If the Higgs boson is not fully reconstructable (e.g., in $H \rightarrow W^{(*)} W^{(*)} \rightarrow 2 \ell 2 v$ ) Higgs off-shell contributions have to be taken into account (which is straightforward at NLO).

At the FCC-ee with $\sqrt{s}=240 \mathrm{GeV}$ (or other $e^{+} e^{-}$machines near this center-of-mass energy), the Higgs-boson production cross-section is strongly dominated by $e^{+} e^{-} \rightarrow Z H$, and $e^{+} e^{-} \rightarrow \nu \bar{v} H$ contributes less than $20 \%[1,35]$. For these two processes, full one-loop corrections in the SM are available [36,37]. For the dominating $Z H$ production mode they are found at the level of $\sim 5-10 \%$. Leading two-loop corrections to this cross section were evaluated at $\mathcal{O}\left(\alpha \alpha_{s}\right)$ [38,39], which were found to amount to $\sim 1-2 \%$. This number has to be compared to the anticipated experimental accuracy of $0.4 \%$ [1-3]. It becomes clear that with a full two-loop calculation of $e^{+} e^{-} \rightarrow Z H$ the intrinsic uncertainty will be sufficiently small. Calculational techniques for $2 \rightarrow 2$ processes at the two-loop level exist, and it is reasonable to assume that, if required, this calculation within the SM can be incorporated for the FCC-ee Higgs precision studies (see also [40] for recent progress in this direction).

For WBF production, the calculation of the full two-loop corrections will be significantly more difficult, since this is a $2 \rightarrow 3$ process. However, one may assume that a partial 
result based on diagrams with closed light-fermion loops and top-quark loops (in a large$m_{t}$ approximation) can be achieved, which should reduce the intrinsic theory uncertainty to below the $1 \%$ level. Given the fact that the WBF process is less crucial than the $H Z$ channel for the Higgs physics program FCC-ee with $\sqrt{s}=240 \mathrm{GeV}$, this will probably be adequate for most practical purposes. It would be desirable to have a complete $2 \rightarrow 4$ calculation at two loops, including both processes with their interference, but very likely factorized processes with on-shell projections will be sufficient.

\subsection{Higgs-boson decay}

The current intrinsic and parametric uncertainties for the various Higgs-boson decay widths are given in Table 2 (see [4]). The status of the intrinsic uncertainties was evaluated following [41-44].

Also the parametric uncertainties can play a non-negligible role for the evaluation of the partial widths. The most important parameters are the bottom quark mass and the strong coupling constant. In Ref. [42] the current uncertainties of $\alpha_{s}$ and $m_{b}$ have been assumed to be $\delta \alpha_{s}=0.0015$ and $\delta m_{b}=0.03 \mathrm{GeV}$. Additionally, $\delta m_{c}=0.025 \mathrm{GeV}, \delta m_{t}=0.85 \mathrm{GeV}$ and $\delta M_{H}=0.24 \mathrm{GeV}$ [45] have been taken into account. The effect on the various partial widths has been evaluated as in Ref. [41] and is shown in the three right columns of Table 2.

When comparing the combined intrinsic and parametric uncertainties with the target precision of FCC-ee [1-3], see the last column in Table 3, it is clear that improvements are necessary. Concerning the intrinsic theory uncertainty, the available predictions for the $f \bar{f}$ and $\gamma \gamma$ channels are already sufficiently precise to match the expected FCC-ee experimental uncertainty. With available calculational techniques, the evaluation of complete two-loop corrections to $H \rightarrow f \bar{f}$ can be achieved. This would reduce the uncertainty of the electroweak contributions to less than $0.1 \%$. Similarly, the complete NLO corrections to $H \rightarrow Z \gamma$ can be carried out with existing methods, resulting in an estimated precision of about $1 \%$. More theoretical work is needed for $H \rightarrow W W, Z Z, g g$, which are currently limited by QCD uncertainties. For $H \rightarrow g g$, the calculation of massless four-loop QCD diagrams will be required, which may be within reach [46], reducing the intrinsic uncertainties to the level of about $1 \%$. For $H \rightarrow W W, Z Z$, the required QCD corrections are essentially identical to those for $e^{+} e^{-} \rightarrow W W$, and it is straightforward to improve them to a practically negligible level. Further significant progress would require the calculation of two-loop electroweak corrections, which for a $1 \rightarrow 4$ process is beyond reach for the forseeable future. Here it should be noted, however, that the $H Z Z$ coupling will be mostly constrained by the measurement of the $e^{+} e^{-} \rightarrow H Z$ production process at FCC-ee with $\sqrt{s}=240 \mathrm{GeV}$, rather than the decay $H \rightarrow Z Z^{*}$, see the discussion in Sect. 4.1, leading to a remaining intrinsic uncertainty of less than $0.3 \%$.

Also shown in Table 3 are the projected parametric uncertainties, assuming FCC-ee precisions. For inputs, we use $\delta \alpha_{s}=0.0002$ and $\delta m_{t}=50 \mathrm{MeV},{ }^{3} \delta M_{H} \sim 10 \mathrm{MeV}$, and $\delta m_{b} \sim 13 \mathrm{MeV}$ and $\delta m_{\mathrm{c}} \sim 7 \mathrm{MeV}$, see [4] for details. ${ }^{4}$ It becomes clear that the intrinsic and parametric uncertainties will be able to match the high anticipated experimental accuracy at FCC-ee.

\footnotetext{
3 This assumes a precision from an $e^{+} e^{-}$top threshold scan. At the highest FCC-ee energy, $365 \mathrm{GeV}$, the top Yukawa coupling can also be inferred from the top threshold dependence on electroweak loop corrections.

4 Note that the numbers in Table 3 do not take into account correlations between the uncertainties in the Higgs production and decay processes or between different decay processes, in particular, entering via $\Gamma_{\text {tot }}$. Their impact can only be evaluated when the full experimental correlation matrix is known, see the discussion in [4].
} 


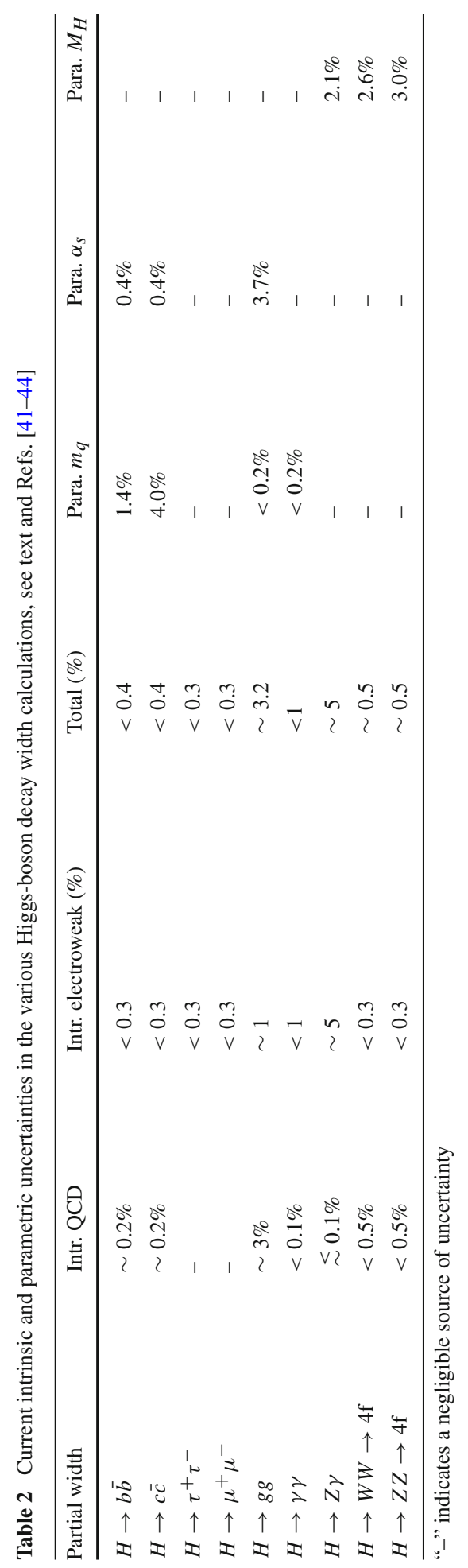




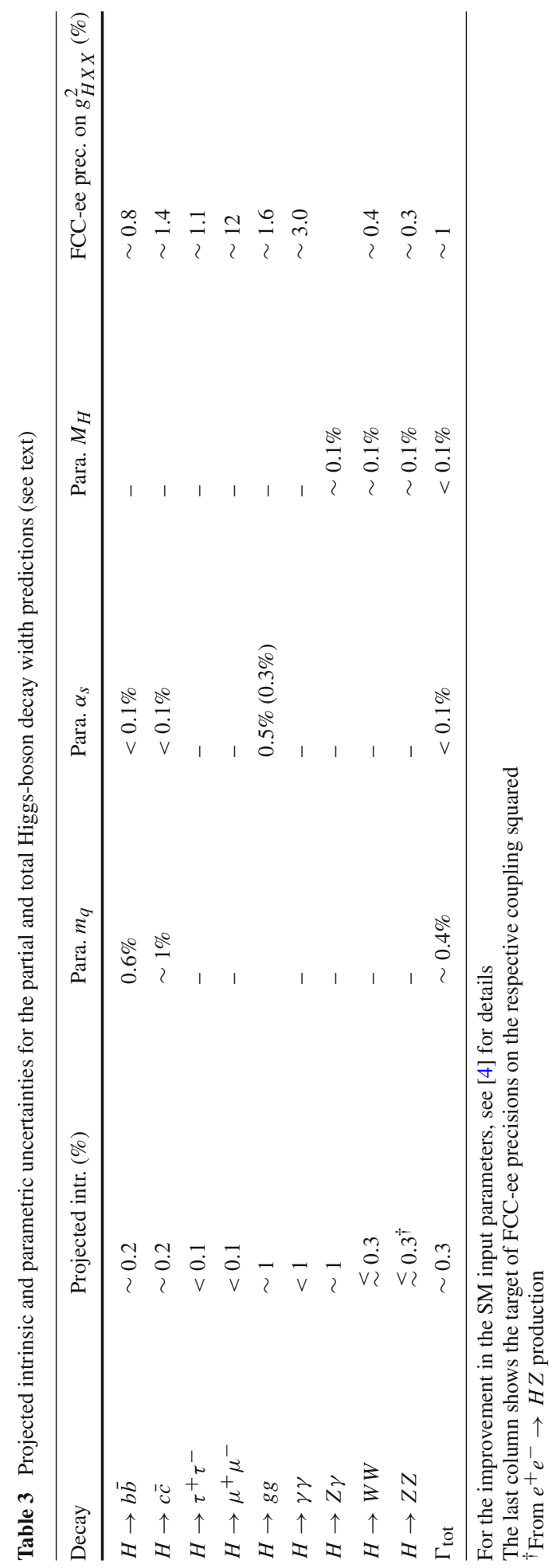




\section{Monte Carlo and exclusive predictions}

To determine systematic uncertainties, to properly extract parameters from the Higgs measurements and to find deviations from SM predictions, it is indispensable to have Monte Carlo (MC) simulations. The challenge compared to LEP/SLC times is the strongly increased experimental precision and much higher luminosities which demands much better simulations. The precise description of QED effects, both regarding the correct normalization of cross sections as well as exclusive multi-photon radiation will be one of the highest priorities. In the recent years, there has been steady progress in dealing with the calculation of higher logarithmic and finite orders in the QED radiation, enhanced by the very small electron mass, see Refs. [47-51]. These very advanced calculations are only partially applicable for the exclusive description of photon radiation which is necessary if cuts are to be applied on those photons. At the level of LO matrix elements, there is a matching procedure between explicit matrix element photons and the electron PDFs in collinear factorization in the context of WHIZARD (cf. below) which has been recently shown to work unexpectedly well [52,53].

From the LEP era, there are many dedicated MC programs which feature soft photon resummation in the exclusive form. Most of them YFS2 / KORALZ [54], BHLUMI [55], BHWIDE [56], YFSWW [57] are based directly on the Yennie-Frautschi-Suura (YSF) resummation scheme [58]. A more powerful coherent exclusive exponentiation (CEEX) at the amplitude level was introduced in [29] and is so far implemented only in KKMC [59] program for two-fermion processes $e^{+} e^{-} \rightarrow f \bar{f}, f=\mu, \tau, v, q$. A generic semiautomatic implementation of CEEX for a wider class of processes would be a very desirable development, and might pave the path towards a consistent inclusion of the non-soft higher-order contributions over the complete photon phase space, merging together genuine electroweak corrections with QED correction calculated to much higher orders, inclusion of spin correlations, initialfinal state interferences and more. The ultimate goal is to match non-soft QED corrections up to fourth order and genuine SM electroweak corrections up to second order.

Multi-purpose event generators allow to simulate the full spectrum of SM (and BSM) processes at FCC-ee. They provide (for the moment) a lower level of precision for the twofermion processes, but allow to simulate them in the same framework as four-, six-, and event eight-fermion processes. Due to the much higher precision and better detectors than at SLC/LEP these processes have to be included already at the $250 \mathrm{GeV}$ stage, and not only if triple electroweak resonances or top pairs are kinematically accessible. In this context a lot of experience has been gained from the large full SM event samples generated for ILC, CLIC and CEPC using WHIZARD [60,61]. This supports leading-logarithmic QED collinear factorization for QED photons, automated NLO QCD corrections for arbitrary processes, while NLO electroweak corrections are under way. For experimental studies, crucial observables are the correct numbers of neutral and charged hadrons. These can only be correctly simulated if the color-flow assignments are correctly made, which includes that the invariant masses of the shower systems from the most prominent processes, $e^{+} e^{-} \rightarrow W^{+} W^{-}, Z Z$ are preserved; in a full electroweak matrix element they are, however, quantum mechanically entangled. The MC hence needs to determine the correct probability of the resonant subprocesses and hand them over to parton shower and hadronization $[62,63]$. It is important to note that the solution of the long-standing problem of the soft photon resummation to narrow width semi-stable charged resonances like $W^{ \pm}$was recently outlined in Ref. [64]. It is also very important to take photon-induced backgrounds from Weizsäcker-Williams QED splittings into account, particularly $\gamma \gamma \rightarrow$ hadrons. WHIZARD has a special treatment for the top threshold run [65] that is used by the FCC-ee collaboration. It also supports all different 
kinds of beam spectra, from simple parameterized files, over Gaussian beam spreads to a sophisticated beamstrahlung setup for linear and circular lepton machines [66].

To search for deviations in the Higgs sector from the SM, WHIZARD supports a large setup of BSM models like supersymmetry, extended Higgs models like 2HDM, Higgs singlet extensions, SMEFT, composite and Little Higgs models, which can be generically extended to its interface to automated Lagrangian model tools, cf., e.g., [67,68]

\section{Outlook and deliverables}

The most important steps to be taken in the future to match the high anticipated accuracy of FCC-ee in the context of Higgs physics and EWPO with SM theory predictions are the following.

- Improved unfolding techniques to go from observables to pseudo-observables.

- Calculations for the EWPO: complete $\mathcal{O}\left(\alpha \alpha_{s}^{2}\right)$ corrections, fermionic $\mathcal{O}\left(\alpha^{2} \alpha_{s}\right)$ corrections, double-fermionic $\mathcal{O}\left(\alpha^{3}\right)$ corrections, and leading four-loop corrections enhanced by the top Yukawa coupling.

- To extract the $W$-boson mass: complete 2-loop corrections to $e^{+} e^{-} \rightarrow W^{+} W^{-}$and to $W \rightarrow f \bar{f}^{\prime}$ (based on the effective field theory framework).

- Development on matching scheme between EW corrections and radiated/resummend photons.

- Full two-loop corrections for $e^{+} e^{-} \rightarrow Z H$ and $e^{+} e^{-} \rightarrow V V H$

- Full two-loop corrections for $H \rightarrow f f(+\gamma)$.

- (Mostly) automatized MC generation including NLO QCD and EW corrections.

- Improvements by roughly one order of magnitude in the determination of the most important SM parameters $\left(m_{t}, m_{b}, \alpha_{s}, \Delta \alpha_{\text {had }}, M_{H}, \ldots\right)$

- Complete simulations of relevant SM processes at different energy stages in full detector simulation

Finally, the following should be kept in mind. The SM constitutes the model in which highest theoretical precision for the predictions of EWPO and Higgs-boson observables can be obtained. This concerns their prediction as well as their extraction. However, as soon as BSM physics will be discovered, an evaluation of the predictions of the EWPO and the Higgs-boson observables in any preferred BSM model will be necessary. The corresponding theory uncertainties, both intrinsic and parametric, can then be larger (see, e.g., [19,69] for the Minimal Supersymmetric SM). A dedicated theory effort (beyond the SM) would be needed in this case.

Acknowledgements The work of S.H. is supported in part by the MEINCOP Spain under contract PID2019110058GB-C21 and in part by the AEI through the Grant IFT Centro de Excelencia Severo Ochoa SEV2016-0597. S.J. acknowledges funding from the European Union's Horizon 2020 research and innovation programme under Grant Agreement No. 951754 and support of the National Science Centre, Poland, Grant No. 2019/34/E/ST2/00457. JRR acknowledges the support by the Deutsche Forschungsgemeinschaft (DFG, German Research Association) under Germany's Excellence Strategy-EXC 2121 “Quantum Universe”-39083330. The authors would like to thank Tord Riemann, who passed away as this report was written, for his decade-long work on EW precision physics for $e^{+} e^{-}$colliders.

Funding Open Access funding provided thanks to the CRUE-CSIC agreement with Springer Nature. This project is co-funded from the European Union's Horizon 2020 research and innovation programme under grant agreement No 95174. 
Open Access This article is licensed under a Creative Commons Attribution 4.0 International License, which permits use, sharing, adaptation, distribution and reproduction in any medium or format, as long as you give appropriate credit to the original author(s) and the source, provide a link to the Creative Commons licence, and indicate if changes were made. The images or other third party material in this article are included in the article's Creative Commons licence, unless indicated otherwise in a credit line to the material. If material is not included in the article's Creative Commons licence and your intended use is not permitted by statutory regulation or exceeds the permitted use, you will need to obtain permission directly from the copyright holder. To view a copy of this licence, visit http://creativecommons.org/licenses/by/4.0/.

\section{References}

1. TLEP Design Study Working Group Collaboration, M. Bicer et. al., First Look at the Physics Case of TLEP. JHEP 01 (2014) 164. arXiv:1308.6176

2. F.C.C. Collaboration, A. Abada et al., FCC-ee: The lepton collider. Eur. Phys. J. Spec. Top. 228(2), 261-623 (2019)

3. FCC Collaboration, A. Abada et al., FCC Physics opportunities. Eur. Phys. J. C 79(6), 474 (2019)

4. A. Freitas et. al., Theoretical uncertainties for electroweak and Higgs-boson precision measurements at FCC-ee (2019). arXiv:1906.0537

5. Two Fermion Working Group Collaboration, M. Kobel et al., Two-Fermion production in electronpositron collisions, in Proceedings, Monte Carlo Workshop: Report of the working groups on precision calculation for LEP-2 physics: CERN, Geneva, Switzerland, March 12-13, June 25-26, October 12-13 Oct 1999, p. 2000. hep-ph/0007180

6. S. Jadach et al., Event generators for Bhabha scattering, in CERN Workshop on LEP2 Physics (followed by 2nd meeting, 15-16 Jun 1995 and 3rd meeting 2-3 Nov 1995) Geneva, Switzerland, February 2-3, 1995, pp. 229-298, 1996. hep-ph/9602393

7. W. Beenakker et al., $W W$ cross-sections and distributions, in CERN Workshop on LEP2 Physics (followed by 2nd meeting, 15-16 Jun 1995 and 3rd meeting 2-3 Nov 1995) Geneva, Switzerland, February 2-3, 1995, pp. 79-139, 1996. hep-ph/9602351

8. C. Chen, X. Mo, M. Selvaggi, Q. Li, G. Li, M. Ruan, X. Lou, Fast simulation of the CEPC detector with Delphes. arXiv:1712.0951

9. CEPC Collaboration, CEPC Software Framework, May, 2018. webpage: http://cepcsoft.ihep.ac.cn/

10. ILD/SiD Collaboration, ILC DBD samples, Sept., 2012. webpage: https://ilcsoft.desy.de/data_samples

11. LCC Collaboration, M. Berggren, Generating the full SM at linear colliders, PoS ICHEP2020 (2021) 903. arXiv:2105.0404

12. H. Ono, A. Miyamoto, Status of ILD new $250 \mathrm{GeV}$ common MC sample production, in International Workshop on Future Linear Colliders, 5 (2021). arXiv:2105.0604

13. C. Potter, SiD Simulation \& Analysis for ILC Snowmass Physics LoIs, in International Workshop on Future Linear Colliders, 6, 2021. arXiv:2106.0081

14. A. Tsaregorodtsev et al., DIRAC: A community grid solution. J. Phys: Conf. Ser. 119, 062048 (2008)

15. CLIC detector, Physics study Collaboration, C. Grefe, S. Poss, A. Sailer, A. Tsaregorodtsev, ILCDIRAC, a DIRAC extension for the Linear Collider community. J. Phys. Conf. Ser. 513, 032077 (2014)

16. M. Frank, F. Gaede, M. Petric, A. Sailer, AIDASoft/DD4hep: v01-17-00, June, 2021. http://dd4hep.cern. $\mathrm{ch} /$

17. D. Yu. Bardin, M. Grunewald, G. Passarino, Precision calculation project report, (1999) hep-ph/9902452

18. ALEPH, DELPHI, L3, OPAL, SLD, LEP Electroweak Working Group, SLD Electroweak Group, SLD Heavy Flavour Group Collaboration, S. Schael et al., Precision electroweak measurements on the $Z$ resonance. Phys. Rept. 427, 257-454 (2006). hep-ex/0509008

19. S. Heinemeyer, W. Hollik, G. Weiglein, Electroweak precision observables in the minimal supersymmetric standard model. Phys. Rept. 425, 265-368 (2006). hep-ph/0412214

20. S. Jadach, M. Skrzypek, QED challenges at FCC-ee precision measurements. Eur. Phys. J. C 79(9), 756 (2019). arXiv:1903.0989

21. A. Blondel et al., Standard Model Theory for the FCC-Ee Tera-Z Stage, in Mini Workshop on Precision EW and QCD Calculations for the FCC Studies?: Methods and Techniques CERN, Geneva, Switzerland, January 12-13 (CERN, Geneva, 2019). arXiv:1809.0183

22. DELPHI, OPAL, ALEPH, L3 Collaboration, S. Schael et al., Electroweak measurements in electronpositron collisions at W-Boson-pair energies at LEP. Phys. Rept. 532, 119-244 (2013). arXiv:1302.3415

23. A. Freitas, Numerical multi-loop integrals and applications. Prog. Part. Nucl. Phys. 90, 201-240 (2016). arXiv: 1604.0040 
24. M. Awramik, M. Czakon, A. Freitas, G. Weiglein, Precise prediction for the W boson mass in the standard model. Phys. Rev. D 69, 053006 (2004). hep-ph/0311148

25. M. Awramik, M. Czakon, A. Freitas, G. Weiglein, Complete two-loop electroweak fermionic corrections to $\sin ^{2} \theta_{\text {eff }}^{\text {lept }}$ and indirect determination of the Higgs boson mass. Phys. Rev. Lett. 93, 201805 (2004). hep-ph/0407317

26. A. Freitas, Higher-order electroweak corrections to the partial widths and branching ratios of the $\mathrm{Z}$ boson. JHEP 04, 070 (2014). arXiv:1401.2447

27. I. Dubovyk, A. Freitas, J. Gluza, T. Riemann, J. Usovitsch, Complete electroweak two-loop corrections to Z boson production and decay. Phys. Lett. B 783, 86-94 (2018). arXiv:1804.1023

28. A. Freitas, Electroweak precision tests in the LHC era and Z-decay form factors at two-loop level, in 12th DESY Workshop on Elementary Particle Physics: Loops and Legs in Quantum Field Theory, p. 6, 2014. arXiv:1406.6980

29. S. Jadach, B.F.L. Ward, Z. Was, Coherent exclusive exponentiation for precision Monte Carlo calculations. Phys. Rev. D 63, 113009 (2001). hep-ph/0006359

30. P. Azzurri, et al., Physics Behind Precision (2017). arXiv:1703.0162

31. A. Denner, S. Dittmaier, M. Roth, L.H. Wieders, Electroweak corrections to charged-current $e^{+} e^{-} \longrightarrow$ 4 fermion processes: Technical details and further results. Nucl. Phys. B 724, 247-294 (2005). hepph/0505042. [Erratum: Nucl.Phys.B 854, 504-507 (2012)]

32. M. Beneke, P. Falgari, C. Schwinn, A. Signer, G. Zanderighi, Four-fermion production near the W pair production threshold. Nucl. Phys. B 792, 89-135 (2008). arXiv:0707.0773

33. S. Actis, M. Beneke, P. Falgari, C. Schwinn, Dominant NNLO corrections to four-fermion production near the W-pair production threshold. Nucl. Phys. B 807, 1-32 (2009). arXiv:0807.0102

34. A. Denner, S. Dittmaier, M. Roth, D. Wackeroth, Electroweak radiative corrections to $e^{+} e^{-}->\mathrm{W} \mathrm{W}$ $\longrightarrow 4$ fermions in double pole approximation: The RACOONWW approach. Nucl. Phys. B 587, 67-117 (2000). hep-ph/0006307

35. A. Arbey et al., Physics at the e+ e- Linear Collider. Eur. Phys. J. C 75(8), 371 (2015). arXiv:1504.0172

36. G. Belanger, F. Boudjema, J. Fujimoto, T. Ishikawa, T. Kaneko, K. Kato, Y. Shimizu, Full one loop electroweak radiative corrections to single Higgs production in e+e-. Phys. Lett. B 559, 252-262 (2003). hep-ph/0212261

37. A. Denner, S. Dittmaier, M. Roth, M.M. Weber, Electroweak radiative corrections to single Higgs boson production in e+ e- annihilation. Phys. Lett. B 560, 196-203 (2003). hep-ph/0301189

38. Y. Gong, Z. Li, X. Xu, L.L. Yang, X. Zhao, Mixed QCD-EW corrections for Higgs boson production at $e^{+} e^{-}$colliders. Phys. Rev. D 95(9), 093003 (2017) arXiv:1609.0395

39. Q.-F. Sun, F. Feng, Y. Jia, W.-L. Sang, Mixed electroweak-QCD corrections to $e^{+} e^{-} \rightarrow H Z$ at Higgs factories, Phys. Rev. D 96(5), 051301 (2017). arXiv:1609.0399

40. Q. Song, A. Freitas, On the evaluation of two-loop electroweak box diagrams for $e^{+} e^{-} \rightarrow H Z$ production. JHEP 04, 179 (2021). arXiv:2101.0030

41. G.P. Lepage, P.B. Mackenzie, M.E. Peskin, Expected Precision of Higgs Boson Partial Widths within the Standard Model (2014). arXiv:1404.0319

42. LHC Higgs Cross Section Working Group Collaboration, D. de Florian et. al., Handbook of LHC Higgs Cross Sections: 4. Deciphering the Nature of the Higgs Sector, 2/2017 (2016). arXiv:1610.0792

43. D. de Florian, et al., LHC Higgs Cross Section Working Group (2016)

44. A. Denner, S. Heinemeyer, I. Puljak, D. Rebuzzi, M. Spira, Standard model Higgs-Boson branching ratios with uncertainties. Eur. Phys. J. C 71, 1753 (2011). arXiv:1107.5909

45. Particle Data Group Collaboration, P.A. Zyla et. al., Review of Particle Physics, PTEP 2020 8, 083 C01 (2020)

46. P.A. Baikov, K.G. Chetyrkin, J.H. Kuhn, Scalar correlator at O(alpha(s)**4), Higgs decay into b-quarks and bounds on the light quark masses. Phys. Rev. Lett. 96, 012003 (2006). hep-ph/0511063

47. J. Ablinger, J. Blümlein, A. De Freitas, K. Schönwald, Subleading Logarithmic QED Initial State Corrections to $e^{+} e^{-} \rightarrow \gamma^{*} / Z^{0^{*}}$ to $O\left(\alpha^{6} L^{5}\right)$. Nucl. Phys. B 955, 115045 (2020). arXiv:2004.0428

48. J. Blümlein, A. De Freitas, C. Raab, K. Schönwald, The $O\left(\alpha^{2}\right)$ initial state QED corrections to $e^{+} e^{-} \rightarrow$ $\gamma^{*} / Z_{0}^{*}$. Nucl. Phys. B 956, 115055 (2020). arXiv:2003.1428

49. J. Blümlein, A. De Freitas, K. Schönwald, The QED initial state corrections to the forward-backward asymmetry of $e^{+} e^{-} \rightarrow \gamma^{*} / Z^{0 *}$ to higher orders. Phys. Lett. B 816, 136250 (2021). arXiv:2102.1223

50. S. Frixione, Initial conditions for electron and photon structure and fragmentation functions. JHEP 11, 158 (2019). arXiv: 1909.0388

51. V. Bertone, M. Cacciari, S. Frixione, G. Stagnitto, The partonic structure of the electron at the next-toleading logarithmic accuracy in QED. JHEP 03, 135 (2020). arXiv:1911.1204

52. J. Kalinowski, W. Kotlarski, P. Sopicki, A.F. Zarnecki, Simulating hard photon production with WHIZARD. Eur. Phys. J. C 80(7), 634 (2020). arXiv:2004.1448 
53. P. Sopicki, J. Kalinowski, W. Kotlarski, K. Mekała, A.F. Zarnecki, Simulating hard photon production with WHIZARD. PoS ICHEP 2020, 285 (2021)

54. S. Jadach, B.F.L. Ward, Z. Was, The Monte Carlo program KORALZ, for the lepton or quark pair production at LEP/SLC energies: From version 4.0 to version 4.04. Comput. Phys. Commun. 124, 233237 (2000). hep-ph/9905205

55. S. Jadach, W. Placzek, E. Richter-Was, B.F.L. Ward, Z. Was, Upgrade of the Monte Carlo program BHLUMI for Bhabha scattering at low angles to version 4.04. Comput. Phys. Commun. 102, 229-251 (1997)

56. S. Jadach, W. Placzek, B.F.L. Ward, BHWIDE 1.00: O(alpha) YFS exponentiated Monte Carlo for Bhabha scattering at wide angles for LEP-1/SLC and LEP-2, Phys. Lett. B390 (1997) 298-308, hep-ph/9608412. also hep-ph/9608412; The Monte Carlo program BHWIDE is available from http://cern.ch/placzek

57. W. Placzek, S. Jadach, Multiphoton radiation in leptonic W boson decays. Eur. Phys. J. C 29, 325-339 (2003). [hep-ph/0302065]

58. D.R. Yennie, S.C. Frautschi, H. Suura, The infrared divergence phenomena and high-energy processes. Ann. Phys. 13, 379-452 (1961)

59. S. Jadach, B.F.L. Ward, Z. Was, The Precision Monte Carlo event generator KK for two fermion final states in $e^{+} e^{-}$collisions. Comput. Phys. Commun. 130, 260-325 (2000). [hep-ph/9912214]

60. M. Moretti, T. Ohl, J. Reuter, O’Mega: An Optimizing matrix element generator (2001). hep-ph/0102195

61. W. Kilian, T. Ohl, J. Reuter, WHIZARD: simulating multi-particle processes at LHC and ILC. Eur. Phys. J. C 71, 1742 (2011). arXiv:0708.4233

62. T. Sjostrand, S. Mrenna, P.Z. Skands, PYTHIA 6.4 Physics and Manual. JHEP 05, 026 (2006). hep$\mathrm{ph} / 0603175$

63. T. Sjöstrand, S. Ask, J.R. Christiansen, R. Corke, N. Desai, P. Ilten, S. Mrenna, S. Prestel, C.O. Rasmussen, P.Z. Skands, An introduction to PYTHIA 8.2. Comput. Phys. Commun. 191, 159-177 (2015). arXiv: 1410.3012

64. S. Jadach, W. Płaczek, M. Skrzypek, QED exponentiation for quasi-stable charged particles: the $e^{-} e^{+} \rightarrow$ $W^{-} W^{+}$process. Eur. Phys. J. C 80(6), 499 (2020) arXiv: 1906.0907

65. F. Bach, B.C. Nejad, A. Hoang, W. Kilian, J. Reuter, M. Stahlhofen, T. Teubner, C. Weiss, Fullydifferential top-pair production at a lepton collider: from threshold to continuum. JHEP 03, 184 (2018). arXiv: 1712.0222

66. T. Ohl, CIRCE version 1.0: Beam spectra for simulating linear collider physics. Comput. Phys. Commun. 101, 269-288 (1997). hep-ph/9607454

67. N.D. Christensen, C. Duhr, B. Fuks, J. Reuter, C. Speckner, Introducing an interface between WHIZARD and FeynRules. Eur. Phys. J. C 72, 1990 (2012). arXiv:1010.3251

68. C. Degrande, C. Duhr, B. Fuks, D. Grellscheid, O. Mattelaer, T. Reiter, UFO-The Universal FeynRules Output. Comput. Phys. Commun. 183, 1201-1214 (2012). arXiv:1108.2040

69. A. Freitas, K. Hagiwara, S. Heinemeyer, P. Langacker, K. Moenig, M. Tanabashi, G.W. Wilson, Exploring Quantum Physics at the ILC, in Community Summer Study 2013: Snowmass on the Mississippi, p. 7, 2013. arXiv:1307.3962 